This must finally put to rest the

lingering perception that BDJs lie

stacked and unopened in the corners of

dental surgeries throughout the land.

\title{
BDJ Myths Shattered
}

Once every three to four years the $B D J$ carries out a readership survey, and the results of the latest one (completed in November 2002) have just been published. A full report will be published in the $B D J$ in a future issue, but for now I thought I would highlight a few of the findings that I found interesting, especially as I believe they disprove some of the common myths and misconceptions about the $B D J$ that still lurk in certain parts of the dental consciousness.

As before, the survey was carried out by the Research Unit of the Policy Directorate at the British Dental Association and is completely independent of the $B D J$. The only link I had with the research was to suggest amendments to some of the questions. The questionnaire was sent to a random sample of 1,000 BDA members (excluding students, overseas and retired members) and was thus similar to the previous readership surveys completed in 1992, 1995, 1997 and 1999. The response was 59\%, similar to previous surveys and quite amazing when compared with readership survey responses in general (which are often below 10\%), thus high enough to limit non-response bias considerably. In other words, the method and response of the survey confirms that the results are as accurate as the research papers published in the $B D J$ itself.

It is important to appreciate this level of accuracy and the confidence it inspires in the $B D J$ readership surveys, partly because the results drive future strategy but also because certain misconceptions still exist concerning the $B D J$. For my part I find it gratifying to know that these results are based on a solid foundation of evidence and are thus substantially reliable. As the $B D J$ has absolutely no influence on the findings and the Research Unit at the BDA is completely independent we know that the findings are basically true.

It is always encouraging to know that people read your publication and our latest survey confirms the $B D J$ 's position at the top of the table of all the regular general dental publications for being consistently read. The findings demonstrate that $90 \%$ of people read all or most issues of each BDJ. In other words, more people read the $B D J$ more often than any other publication. This must finally put to rest the lingering perception that $B D J$ s lie stacked and unopened in the corners of the dental surgeries throughout the land.

But dental publications are not just about reading for reading's sake - they are about the relevance and usefulness of content. Once again the 'News' section is found to be the most useful, then research summaries followed by case reports. Classified advertisements come fourth, disproving another commonly-held myth that people only read the classified section, although claasified adverts are still fourth in the list of sections found most useful with $81 \%$ of respondents finding them very useful indeed. That is an exceptionally high figure for non-editorial content, demonstrating the diversity of value that readers get from each issue.

Yet another myth that this survey shatters is the apparent disregard for product advertisements. Despite the requests I used to receive to ensure adverts are placed back-to-back (theoretically to allow people to tear them out without damaging the editorial content) it appears people find them genuinely useful. This is seen in the finding that 57\% find advertisements fairly useful, doubtless a welcome result for all the advertisers. Even more gratifying, 83\% (a very high figure) claim to respond to advertisements in the BDJ, which is a much more relevant finding.

For an editor, a readership survey is a scary event, possibly revealing a number of things the editor does not want to know. However, surveys such as this one are far more valuable than the individual comments I receive from people who either approach me or write to me, because the survey reflects the true position. This time, again, it has confirmed the BDJ's leading position in dental publishing and also confirmed that a number of myths that still linger in certain places about the $B D J$ are just that - simply myths.

Mike Grace m.grace@bda-dentistry.org.uk 